\title{
Impact of Incentive Mechanism on Demand Response Programs in Smart Microgrids with Electric Vehicles
}

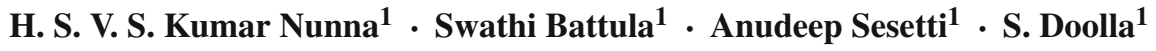

Received: 12 June 2015 / Revised: 28 August 2015 / Accepted: 29 August 2015 / Published online: 10 September 2015

(C) Springer Science+Business Media Singapore 2015

\begin{abstract}
The demand response (DR) potential of plugin electric vehicles can be tapped by designing appropriate DR strategy and incentive mechanisms. In this paper, a new incentive mechanism called priority rewarding is proposed to fairly cater incentives to end-users based on the type of load and their active participation in DR programs. Also as a DR Strategy, bilateral contracts between end-users participating in DR and the owners of distributed generators is obtained using second price auction mechanism. In order to prove the suitability of the proposed incentive mechanism with the DR strategy to microgrid scenario, an agent based energy trading scenario is developed using Java Agent Development Environment. The developed agent system is applied to a case study system with a grid connected microgrid and the obtained results have suggested the successful adoption of the proposed methodology.
\end{abstract}

Keywords Microgrid - Demand response $\cdot$ Plug-in electric vehicle $\cdot$ Incentive mechanism

\section{Introduction}

Demand side management (DSM) programs offer numerous advantages to electrical utilities such as reduced system peak demand, improved plant load factor, system reliability and efficiency, reduced investments in peak power plants etc [1]. Demand response (DR) is one of the DSM programs in which consumers with elastic or low priority loads can participate and shift the load operation to off-peak or low electricity

S. Doolla suryad@iitb.ac.in

1 Department of Energy Science and Engineering, Indian Institute of Technology (IIT) Bombay, Mumbai, India price intervals [2-4]. In the context of microgrids, DR programs assist microgrid operation by shifting the elastic loads to off peak periods, thus maintaining the energy balance [5]. Elastic loads are broadly categorized into adjustable, curtailable and shiftable loads, further shiftable loads are categorized into continuous and discontinuous mode of operation [6]. In order to accommodate various responsive loads into DR programs, a DR mechanism must be capable of providing incentives according to the flexibility offered by them.

Plug-in electric vehicle (PEV) is one such load which is idle most of the day and has huge DR potential [7]. They can be charged in different charging modes such as slow, normal and quick charging modes, from DR perspective they can be categorized as adjustable loads. Therefore, PEVs are considered as responsive loads which can provide solution to renewable intermittency by participating in ancillary markets to provide reserve and energy for a smart distribution system or a microgrid $[8,9]$. The energy resource scheduling of PEVs in an intelligent parking lot consisting of renewable sources is presented considering both economical and technical aspects in [10]. These include provision of energy/ reserve service by PEVs, inclusion of PEV owner preferences and PEV charge/discharge scheduling based on electricity tariff. Uncoordinated charging of PEVs may lead to overloading of distribution system and can create unbalance, and this can be mitigated by time-varying pricing schemes which play a significant role in shifting PEV charging from peak hours to off-peak hours $[11,12]$. Load shifting to low-tariff intervals may cause peak rebound effect, in order to avoid rebound monetary incentives are offered to PEV users for further shifting of PEV load [13]. Financial incentives can also be offered by performing sensitive analysis of the cost savings of EV owners after participating in DR programs [14]. Thus coordinated charging of PEVs can be achieved by providing 
incentives to PEV users which arises the necessity of a suitable incentive mechanism for active PEV participation into DR programs.

In literature, there are several incentive mechanisms available. Reference [15] categorizes DR programs into time based and incentive based DR programs which are applicable in the present context of smart grid. In this paper, it is assumed that time based programs are effective in reforming the load shape and analyzed the impact of incentive based programs for reliability triggered events by defining a suitable DR model. In [16], a dynamic incentive strategy is proposed to motivate electricity consumers to participate in DR through time dependent pricing. In this, monetary incentives are offered by the utility to consumers which is a part of the total benefit gained by the system through DR. Reference [17] presents a coupon incentive-based DR where electricity pricing is flat rate and consumers participating in DR are given financial rebates in the form of voluntary coupon incentives. Utilities offer coupon incentives in peak demand intervals (DI)s where they sense a price spike, consumers voluntarily can shift their demand by accepting the coupon based on the coupon price. Reference [18] presents incentive mechanisms based on the overall benefit gained by the system in purchasing the energy from grid with an assumption of other loads being unchanged, which may not be the case in most of the practical scenarios.

Application of multi-agent system (MAS) in developing energy markets for grid connected microgrid systems with DR techniques has gained popularity owing to its features robustness, flexibility and extensibility $[5,19]$. In the present context, an intelligent framework is needed to incorporate PEVs into DSM programs of microgrids with suitable DR strategy for which MAS is a suitable approach.

The present paper proposes a new incentive mechanism which distinctly captures the flexibilities offered by various DR loads and accordingly caters incentives to end-users to prompt continuous DR participation. As a DR strategy, bilateral contracts have been allotted based on forecast information to schedule elastic loads in surplus power intervals. Second price auction (SPA) mechanism is used for obtaining bilateral contracts rather than continuous double auction (CDA) to reduce auction clearing time which has significant importance in choosing an auction mechanism. The charging of elastic loads is dynamically scheduled by exercising bilateral contracts based on forecast and real time power mismatch without violating their comfort criteria. SPA mechanism is used to obtain bilateral contracts, explained in "Second Price Auction for Bilateral Contracts" section. The proposed priority banking incentive mechanism is detailed in "Priority Rewarding Incentive Mechanism" section. In order to implement the proposed mechanism, a market environment is simulated using MAS, which is described in "Agent Architecture for Demand Response" section. In "Case Study" section, a case study is presented to test the performance of the proposed mechanisms followed by the conclusions.

\section{Second Price Auction for Bilateral Contracts}

Second price auction is derived from the fundamental concept of Vickrey auction mechanism in which bidding one's true value is the dominant winning strategy [20]. In SPA, bidders submit their bids in sealed-bid form simultaneously and the highest bidder wins the auction but pays the second highest bid value. Though it is counter-intuitive that bidders bid their true valuation rather than placing higher bids (greater than true value) in order to win the bid, but there will be no advantage in doing so. This can be justified using the following scenarios.

Let $b_{i}$ be the price bid by $i$ th bidder, $H B$ is the highest bid, $T V$ is the true value, $S B$ is the second highest bid.

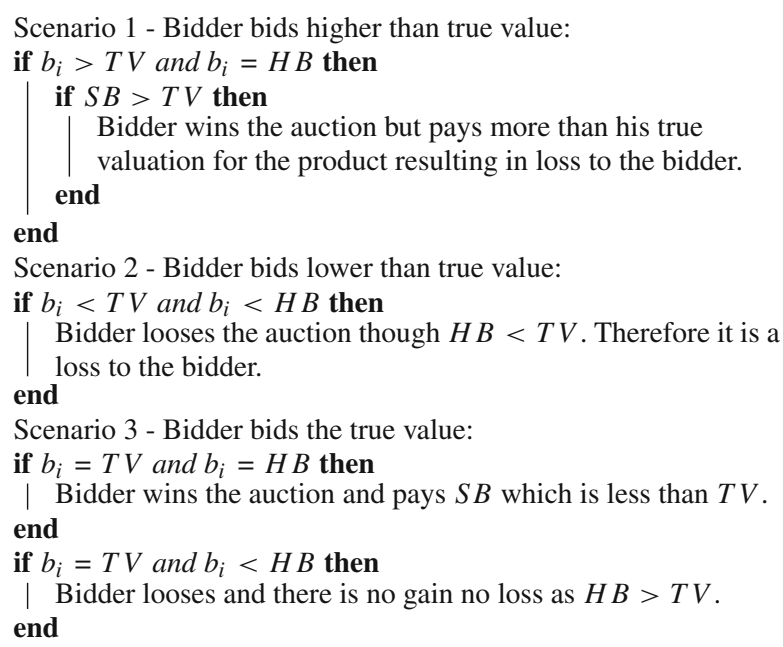

From these scenarios, it can be concluded that the dominant winning strategy is to quote the true valuation of the bidder and thus the market is cleared in single iteration. In the present context, time elapsed while conducting auction is of critical importance and the advantage of using SPA is that it is cleared in single iteration making it suitable in the situations where auction clearing time is critical. In this paper, the SPA mechanism is used to organise auction among Distributed generator (DG)s for obtaining the bilateral contacts for scheduling the operation of elastic loads. DGs submit their individual offers consisting of 'price per $\mathrm{kWh}$, maximum $\mathrm{kW}$ offered' for requested DI. The DG with lowest 'price per $\mathrm{kW}$ ' wins the auction and will be paid the second lowest quote. Bilateral contracts are profitable for DGs in the intervals where electricity prices are low. From SPA point of view, forecasted Market Clearing Price (MCP) values can be treated as the true valuation of DGs, because by obtaining the contracts DGs can make sure that selling price is not less 
than that of the forecast price. Therefore, DGs participate in SPA for bilateral contracts by quoting forecast MCP. DGs are chosen in the order of their 'price per kWh' and are assigned bilateral contracts based on the total elastic load requirement.

\section{Priority Rewarding Incentive Mechanism}

Incentive mechanisms play a notable role in making the DR programs success by fairly motivating the consumers to participate in DR. There are various type of elastic loads to include in DR programs. The flexibility offered by these elastic loads vary based on the type of load, thus these are segregated into shiftable, adjustable and curtailable depending on their amenability in participating DR programs. In order to capture the flexibility offered by various DR loads and drive continuous DR participation of consumers, the following incentive mechanism is proposed.

In this incentive mechanism the electricity consumers those participate in DR programs are assigned an index called Incentive metric (IM). IM takes into account the number of DR enrollments as well as the flexibility offered by the loads. The value of $I M$ for an $i$ th consumer, $I M^{i}$ is calculated as,

$$
I M_{i}^{*}=\frac{E C_{i}-E U_{i}}{\sum_{i=1}^{n} E C_{i}-\sum_{i=1}^{n} E U_{i}}+\frac{F_{i}}{\sum_{i=1}^{n} F_{i}}
$$

and

$$
I M_{i}=\frac{I M_{i}^{*}}{\sum_{i=1}^{n} I M_{i}^{*}}
$$

where $I M_{i}^{*}$ is the incentive metric which has a maximum value of 2 . In order to have a 'unity' cumulated quantity it is normalized as $I M_{i}$, which is the final incentive metric. In (1), $E C_{i}$ is the DR enrollment count of $i$ th consumer in a microgrid and ' $n$ ' is the total number of consumers enrolled in DR program. $E U_{i}$ is a parameter defined to keep track of the incentives utilized by $i$ th consumer which gets cumulated after every interval as,

$E U_{i}=\frac{P_{A}^{i}(t)}{T_{G}(t) \cdot I M_{i}}$

where $P_{A}^{i}(t)$ is the quantity of power in $\mathrm{kW}$ which is allowed to purchase from the microgrid market by $i$ th consumer in interval $t, T_{G}(t)$ is the total available generation in $\mathrm{kW}$ in interval $t$. In (1), $F_{i}$ is a function defined to measure the flexibility offered by $i$ th consumer and is calculated as,
$F_{i}=\sum_{l=1}^{L_{i}} f_{l}$

where $L_{i}$ is the total number of loads participating in DR of $i$ th consumer and $f_{l}$ is the flexibility offered by load $l$ of a consumer participating in DR which is calculated as,

$f_{i}=\left\{\begin{array}{l}1-\left(\frac{O I_{i}-C I_{i}}{P I-B I_{i}+1}\right) \text { if } C I_{i}<O I_{i} \\ 0 \text { if } C I_{i} \geq O I_{i}\end{array}\right.$

where $O I_{i}$ is the total number of operating intervals specified by DR load of $i$ th consumer, $C I_{i}$ is the number of intervals completed or operated, $P I$ is the present interval and $B I_{i}$ is the beginning interval specified by the consumer. The $I M_{i}$ represents the share of $i$ th buyer, $P_{S}^{i}$, in total available generation and is more or less than the required demand, $P_{L}^{i}$ in any interval $t$.

The following cases detail the possible scenarios and explain how the auction process progresses.

Case $1 P_{S}^{i}<P_{L}^{i}$ : In this scenario, $i$ th buyer is allowed to participate in the market for a quantity $P_{A}^{i}=P_{S}^{i}$ and the remaining quantity is to be purchased from the Utility Agent (UA). Here $E U_{i}$ will be equal to 1 .

Case $2 P_{S}^{i}>P_{L}^{i}$ : In this scenario, as the required power is less, $P_{A}^{i}$ will be equal to $P_{L}^{i}$. Here $E U_{i}$ is less than one because $P_{A}^{i}<P_{S}^{i}$.

Consumers with higher $I M$ value will have greater share in local markets and thus the power purchased from utility grid which is at a higher price (compared to local MCP) is less. Once the incentive is utilized, $I M$ is updated by deducting the term $E U_{i}$, eventually $I M$ reaches value 'zero' unless there are additional enrollments. Thus, the proposed incentive mechanism encourages consumers to participate actively in DR programs.

\section{Agent Architecture for Demand Response}

To implement the energy trading scenario in microgrid markets, a suitable agent structure is required consisting mainly two categories of agents:(a) Agents participating in energy auction on behalf of energy sellers and buyers, (b) Agents conducting auction among participants.

The proposed agent architecture for DR including second price auction and priority rewarding incentive mechanism is shown in Fig. 1. 


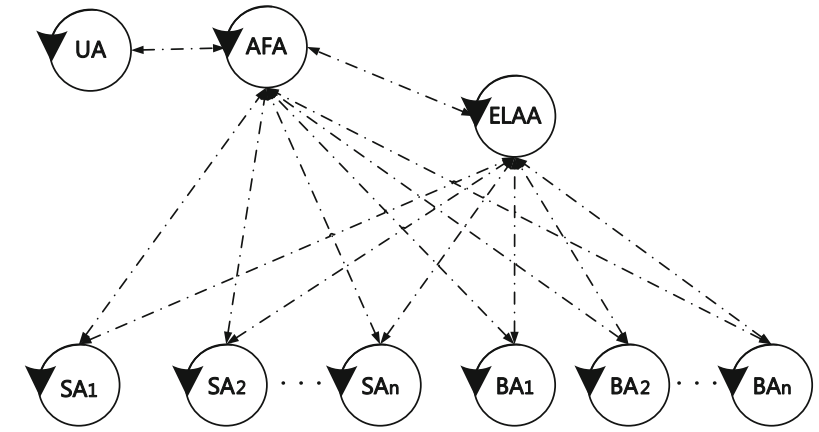

Fig. 1 Agent Hierarchy for PEVs

\section{Role of Agents}

Auction facilitating agent (AFA) and elastic load aggregator agent (ELAA) are auction administering agents, seller agent (SA) and buyer agent (BA) are auction participants representing DG and loads respectively and UA represents utility grid.

SAs and BAs collect generation and load data from respective DGs and loads, the load information gathered by BAs consists of elastic and inelastic components. BAs submit bids for inelastic component to AFA along with SAs to participate in market, while the elastic load information is sent to ELAA. AFA is responsible for conducting auction for locally present SAs and BAs in the microgrid and requesting UA for power inflow or outflow whenever required. AFA uses first price CDA mechanism to conduct auction among SAs and inelastic loads.

\section{Methodology}

The proposed architecture is executed in four stages.

Stage 1 Tentative DR Load Scheduling: In this stage, ELAA collects DR load information from BAs and generates a tentative schedule based on their comfort criteria and forecast surplus power intervals such that the power mismatch $\left(\Delta P_{f}\right)$ is minimal. After generating the DR load schedule, ELAA aggregates DR load for each DI and invites quotes from SAs for the corresponding intervals in order to obtain bilateral contracts. SAs respond by sending an 'offer' value for each DI.

Stage 2 Second Price Auction and Bilateral Contracts for Elastic loads After receiving offers from SAs, ELAA conducts Second Price Auction among the SAs (as discussed in "Second Price Auction for Bilateral Contracts" section) and chooses the SA with minimum 'price per kWh' value. A bilateral contract is made with SAs to serve the DR load upon the request of ELAA. The auction clearing price is the second lowest price as mentioned in "Second Price Auction for Bilateral Contracts" section.

Stage 3 Correction to DR scheduling based on Realtime Data: In each interval, AFA collects the real time generation and load data from SAs and BAs respectively, and submits the power mismatch $\left(\Delta P_{r}\right)$ information to ELAA before conducting CDA among SAs and BAs. When the actual generation and load values deviate from those of predicted, $\Delta P_{r}$ will be different from the $\Delta P_{f}$. In order to have a fair market based on realtime data, ELAA dynamically changes the DR load scheduling. This is done based on $\alpha$ and risk factor (RF) values such that the resulting $\Delta \mathrm{P}$ is minimum. $\alpha$ is defined as the ratio of minimum number of intervals required to serve the elastic/DR load to total number of available intervals. RF is a parameter assigned to depict the reliance on the forecast information and the value chosen is between $\alpha_{\min }(=0)$ and 1. The maximum value of RF chosen is limited to 1 in order to limit $\alpha_{\max }$ value to 1 . This is to make sure the DR load is served before the DL mentioned by user. Higher the value of RF chosen, greater is the dependency on forecast necessitating accurate prediction tools. The steps involved in stage 3 and 4 are shown in Fig. 2.

The following are the possible cases that can occur while rescheduling $\Delta P_{r}$.

Case $1 \Delta P_{f}>0, \Delta P_{r}>0$ and $\alpha<R F$ :

ELAA schedules DR load upto a maximum value of real time $\Delta P_{r}$ and exercises contracts from SAs as per SPA results. The SA which offers lowest 'price per $\mathrm{kWh}$ ' is assigned a bilateral contract from the minimum of the following two choices: (a) The total scheduled DR load b) Maximum quantity of power offered in the current DI. If there is an excess DR load, additional contracts will be assigned to remaining SAs, chosen in the increasing order of their 'price per kWh'.

Case $2 \Delta P_{f}<0, \Delta P_{r}>0$ and $\alpha<R F$ :

As the $\Delta P_{f}$ is negative, no contracts were made by ELAA but real time $\Delta \mathrm{P}$ being positive, a new contract is made for a 'price per kWh' of $11.25 \mathrm{c} / \mathrm{kWh}$, which is the average value of Grid Selling Price (GSP) and Grid Buying Price (GBP). This contract is awarded equally among SAs enrolled for bilateral contracts.

Case $3 \Delta P_{f}>0, \Delta P_{r}<0$ and $\alpha<R F$ :

ELAA doesn't exercise bilateral contract.

Case $4 \alpha>R F$ and for any value of $\Delta P_{f}$ and $\Delta P_{r}$ :

Bilateral contract is exercised following cases 1, 2 and 3, and ELAA participates in the market for the remaining quantity of elastic load. The choice of RF depends on risk attitude of ELAA while scheduling the DR load, higher the value of RF greater the risk involved because there exist a possibility of increase in peak load in further intervals. 


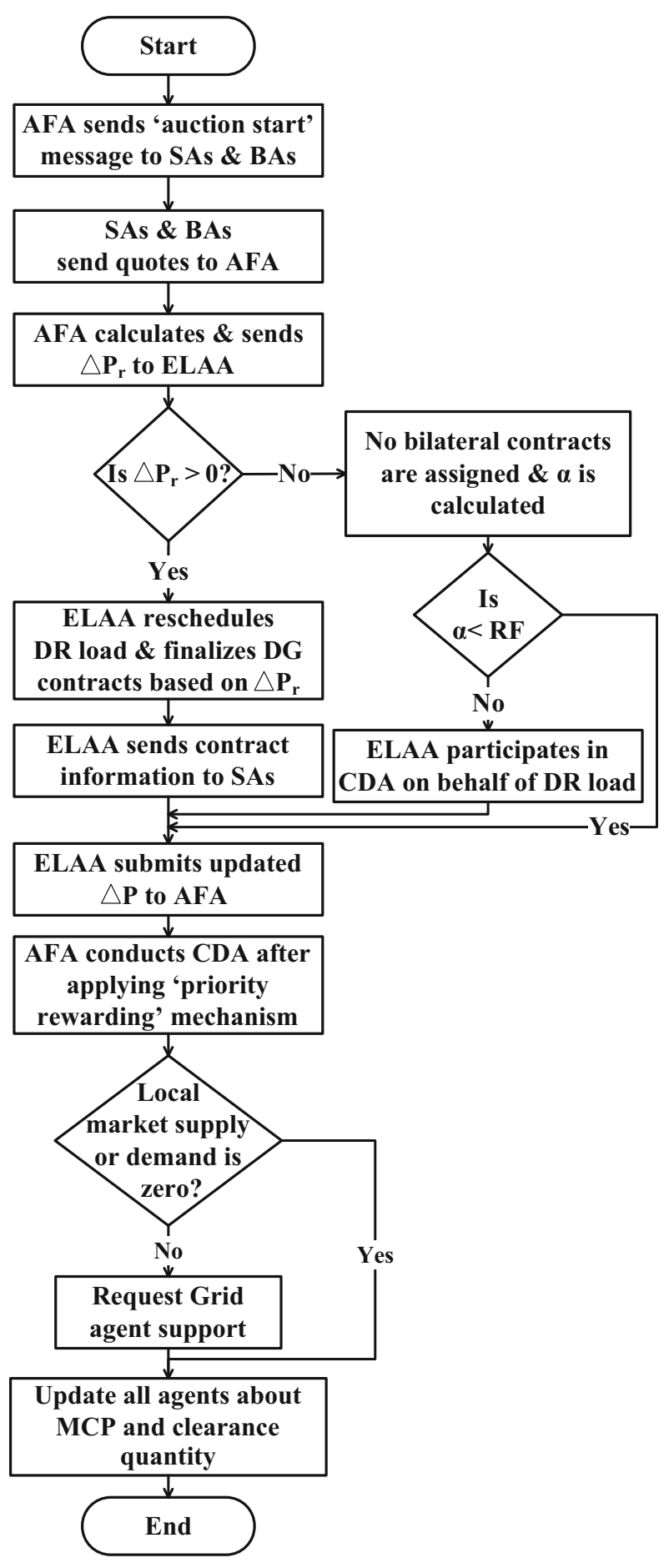

Fig. 2 Auction procedure for stage 3 and 4 in a demand interval DI

At the end of this stage (which is 15 min before the actual schedule), bilateral contracts among elastic loads and SAs are finalized. If there is any elastic load left in this stage, ELAA represents this load and participates in CDA in next stage. Once the bilateral contracts are finalized, ELAA stores successful contract information and communicates it back to SAs and BAs.

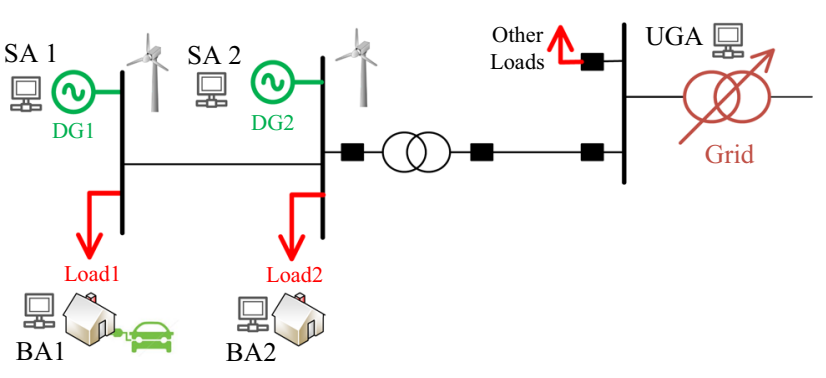

Fig. 3 A case study system with a grid-connected microgrid

Table 1 Comfort criteria of elastic loads participating in DR

\begin{tabular}{lllllll}
\hline Load & BI & EI & OI & kW & Load type & DR category \\
\hline BA11 & 1 & 16 & 4 & 16.8 & PEV & Adjustable \\
BA12 & 1 & 16 & 4 & 16.8 & PEV & Adjustable \\
BA21 & 1 & 16 & 4 & 15 & Pump & Shiftable \\
BA22 & 1 & 16 & 4 & 15 & Pump & Shiftable \\
\hline
\end{tabular}

Stage 4 ELAA submits the updated $\Delta \mathrm{P}$ to AFA, following which AFA conducts CDA after applying priority banking incentive mechanism which is described in "Priority Rewarding Incentive Mechanism" section. At the end of each DI, AFA and ELAA send the generation and load set points to DGs and loads.

\section{Case Study}

In order to illustrate the impact of the proposed incentive mechanism and pricing method on consumer participation in DR programs, a grid connected microgrid system shown in Fig. 3 is considered.

The case study system has two DGs, each rated $100 \mathrm{~kW}$ and two connected loads of $100 \mathrm{~kW}$ each. PEVs and water lifting pumps are taken as elastic loads and the details of these loads and their operating criteria are given in Tables 1 and 2 . The charging efficiency of these loads is assumed to be $90 \%$.

The reason for choosing similar operating criteria for PEV and pump loads is to demonstrate the effectiveness of the proposed incentive mechanism, in encouraging consumers to participate in DR by quantifying the flexibility offered by various type of loads. In this case study, the market price limits are set as 9 and $13.5 \mathrm{c} / \mathrm{kWh}$ and in case of balanced supply and demand the MCP is $11.25 \mathrm{c} / \mathrm{k}$ Wh.

Table 3 presents generation forecast, load forecast and supply-demand mismatch $\left(\Delta P_{f}\right)$ data along with $\mathrm{MCP}$ (assumed to follow previous day trading) and energy sell- 
Table 2 Charging modes of Tesla Roadster [21]

\begin{tabular}{lrr}
\hline Mode & $\mathrm{kW}$ & $\mathrm{hr}$ \\
\hline Slow & 1.8 & 48 \\
Normal & 9.6 & 9 \\
Quick & 16.8 & 5 \\
\hline
\end{tabular}

Table 3 Generation and load forecast information

\begin{tabular}{|c|c|c|c|c|c|c|c|c|}
\hline \multirow[t]{2}{*}{ DI } & \multicolumn{2}{|c|}{ Sellers ${ }^{\mathrm{a}}$} & \multicolumn{2}{|c|}{ Buyers $^{a}$} & \multirow[t]{2}{*}{$\Delta P_{f}^{a}$} & \multirow[t]{2}{*}{$\mathrm{MCP}$} & \multicolumn{2}{|l|}{ Price $^{b}$} \\
\hline & SA1 & SA2 & BA1 & BA2 & & & SA1 & SA2 \\
\hline 1 & 50 & 50 & 80 & 80 & -60 & 12.30 & 12.30 & 12.33 \\
\hline 2 & 50 & 50 & 90 & 80 & -70 & 12.43 & 12.43 & 12.44 \\
\hline 3 & 50 & 50 & 90 & 90 & -80 & 12.56 & 12.56 & 12.58 \\
\hline 4 & 50 & 60 & 90 & 90 & -70 & 12.35 & 12.35 & 12.37 \\
\hline 5 & 70 & 70 & 70 & 60 & 10 & 11.14 & 11.14 & 10.83 \\
\hline 6 & 60 & 70 & 60 & 50 & 20 & 10.95 & 10.95 & 10.38 \\
\hline 7 & 80 & 80 & 60 & 60 & 40 & 10.69 & 10.27 & 10.25 \\
\hline 8 & 70 & 80 & 60 & 50 & 40 & 10.64 & 10.41 & 10.01 \\
\hline 9 & 70 & 80 & 60 & 60 & 30 & 10.83 & 10.57 & 10.36 \\
\hline 10 & 70 & 80 & 60 & 60 & 30 & 10.83 & 10.57 & 10.36 \\
\hline 11 & 60 & 70 & 70 & 70 & -10 & 11.46 & 11.46 & 11.4 \\
\hline 12 & 60 & 60 & 70 & 70 & -20 & 11.62 & 11.62 & 11.64 \\
\hline 13 & 80 & 90 & 60 & 60 & 50 & 10.56 & 10.17 & 10.02 \\
\hline 14 & 80 & 90 & 60 & 60 & 50 & 10.56 & 10.17 & 10.02 \\
\hline 15 & 70 & 80 & 70 & 70 & 10 & 11.16 & 11.16 & 10.88 \\
\hline 16 & 70 & 70 & 70 & 60 & 10 & 11.14 & 11.14 & 10.83 \\
\hline
\end{tabular}

${ }^{a}$ Unit of power in $\mathrm{kW}$

${ }^{\mathrm{b}}$ Unit of Price in $(\mathrm{c} / \mathrm{kWh})$

ing price (ESP) of SA1 and SA2 per kWh respectively for 16 DIs of 15 min duration each. Table 4 presents the tentative schedule of DR loads generated by ELAA and the aggregated DR potential (from stage 1). From the schedule, it can be observed that adjustable loads offer better flexibility in minimizing the mismatch.

Table 4 also shows the 'offers' placed by SAs to obtain bilateral contracts. It can be noted from Tables 3 and 4 that ELAA invites bids from SAs in surplus power intervals where price quoted by SAs are expected to be relatively low and 'offers' placed by them is the ESP of previous day which is their true value of evaluation for energy. The results of SPA are shown in the last column of Table 4. In this case study, the maximum quantity of power offered by each SA to participate in SPA is limited to $30 \mathrm{~kW}$. Therefore, whenever the required DR load exceeds $30 \mathrm{~kW}$ it will be assigned to SA with next lowest bid. Thus, in intervals 7, 8, 13 and 14 bilateral contracts are assigned to both SA1 and SA2. It can also be observed from Table 4 that the auction winner is SA2 in most of the intervals as the offer placed by SA2 is the lowest.

Table 5 shows real time generation, load data and MCP without DR participation. It also presents the inelastic load

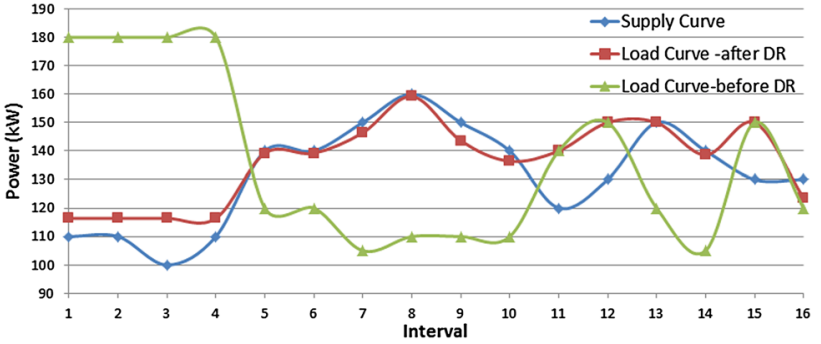

Fig. 4 Generation and demand without and with DR participation

component and $\Delta P_{r}$. Based on the $\Delta P_{r}$, ELAA reschedules DR loads dynamically in order to minimize the resulting $\Delta \mathrm{P}$ in the corresponding interval. Real time scheduling of DR loads and the finalized DR contracts are shown in Table 6. The final schedule generated depends on the $\Delta P_{r}, \alpha$ and RF. In the present case study, RF value is taken as 1 and $\alpha$ value will be less than ' 1 ' because OI of each load is less than the available intervals corresponding to each DI.

Figure 4 shows generation curve and demand curve without and with DR participation, better alignment of load with the generation can be observed with DR participation. Fig. 5 shows scheduling of elastic load before and after DR participation, and it can be depicted that DR load is catered in surplus intervals.

Table 7 presents various index values after applying proposed incentive mechanism and DR model. During intervals 1 to 4 , it can be seen that the IM values for both the loads are same implying equal generation share each $55 \mathrm{~kW}(50 \mathrm{~kW}$ in interval 3) and the remaining load is served by utility grid. The reason for having same IM values is that both loads have same number of enrollments and operating intervals. In further intervals a variation in IM indices can be noted, this is because of the change in flexibilities offered by different DR loads.

In intervals 11 and 12, the IM value of L1 is greater than that of L2 due to greater flexibility offered by the adjustable load L1 compared to L2. This is because the PEVs are scheduled for charging during intervals 6 to 10 according to available power surplus. Due to the adjustable nature of EVs, they can be charged even when the excess available generation is as low as $2 \mathrm{~kW}$ and at the same time they can be charged quickly when larger quantity of excess supply is available. As the IM index of L1 is higher in the intervals 11 and 12, it has higher generation share and is served completely in the microgrid whereas some load of L2 is served by utility grid resulting in relatively higher energy charges. By the end of interval 13, L1 has IM value of ' 1 ' and L2 has 'zero' as the flexibility offered by load L2 is none. Thus L1 has a generation share of $130 \mathrm{~kW}$ whereas $\mathrm{L} 2$ has to purchase power from the grid. The remaining intervals are power surplus intervals where incentive mechanism is not applicable. 


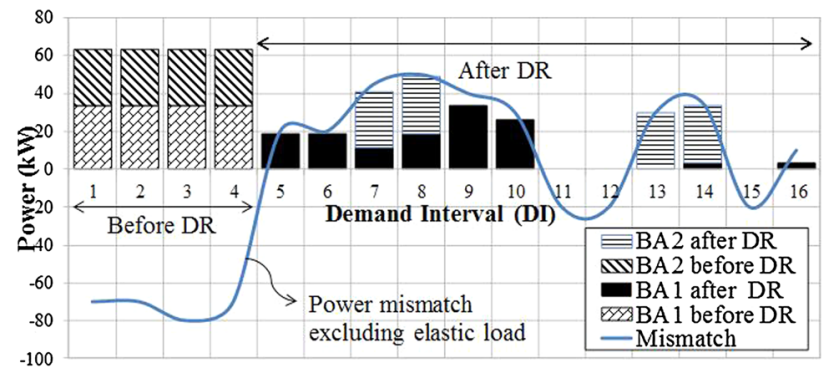

Fig. 5 Scheduling of elastic load before and after DR participation

From local generation share values of loads given in Table 7 , it can be comprehended that the average price of energy purchased by BA2 is greater than that of BA1 due to lesser share of BA2 in local generation compared to BA1. This is because BA1 has adjustable loads that offer relatively more flexibility compared to shiftable loads of BA2.

To summarize, it can be observed that the proposed incentive mechanism is not only able to distinguish different type of loads and benefit them accordingly but also encourage them to continuously participate in DR by forcing them to purchase power from the grid whenever the IM index goes zero. It eventually drives the given system (with rational DGs and responsive end-users) towards the social-equilibrium where the energy price equals to the market equilibrium (supply and demand matches).

The proposed mechanism can be applied in different market environments with little modifications which include re-tuning the auction set-points and price range, and changing the bidding strategy parameters of DGs and loads. The only drawback of the method is that, both being relative quantities they vary independent of each other, the first term of the Eq. (1) may dominate as the cumulative $E U_{i}$ reaches close to $E C_{i}$. Table 8 shows monetary savings gained by elastic loads of BA1 and BA2 by participating in DR.

\section{Comparison with Existing Incentive Mechanisms}

The incentive mechanism presented in this paper is compared against the mechanism proposed in reference [18] where a ranking based incentive mechanism is presented to identify the consumers who are actively involved in DR programs. The ranking is done based on the index given in (6).

$P I(i)=\frac{N_{i}}{\sum_{i=1}^{T} N_{i}}+\frac{P_{i}}{\sum_{i=1}^{T} P_{i}}$

where $P I(i)$ is the priority index of $i$ th consumer, $N_{i}$ is the number of DR enrollments, $P_{i}$ is the sum of the ratings of the elastic loads participating in DR in a day in $\mathrm{kW}$, and $T$ is the total number of consumers in the microgrid.

The above index is evaluated for every DR participation and all the index values are compared for relative raking of the end-users, thus the loads that participate more often and with large rating in DR gets higher rankings. These loads will be given first priority in dispatching the locally available generation.
Table 4 Second price auction \& DG contract allocation (Stage-1 and Stage-2)

\begin{tabular}{|c|c|c|c|c|c|c|c|c|c|c|c|}
\hline \multirow[t]{3}{*}{ DI } & \multirow[t]{3}{*}{$\Delta P_{f}$} & \multicolumn{7}{|c|}{ Tentative DR load schedule } & \multicolumn{3}{|c|}{ Second price auction } \\
\hline & & \multirow[t]{2}{*}{ BA11 } & \multirow[t]{2}{*}{ BA12 } & \multirow[t]{2}{*}{ BA21 } & \multirow[t]{2}{*}{ BA22 } & \multirow[t]{2}{*}{$\Sigma$ DR Load } & \multicolumn{2}{|c|}{ Contract } & \multicolumn{2}{|l|}{ Quote } & \multirow[t]{2}{*}{ Winner } \\
\hline & & & & & & & SA1 & SA2 & SA1 & SA2 & \\
\hline 1 & -60 & 0 & 0 & 0 & 0 & 0 & 0 & 0 & & & \\
\hline 2 & -70 & 0 & 0 & 0 & 0 & 0 & 0 & 0 & & & \\
\hline 3 & -80 & 0 & 0 & 0 & 0 & 0 & 0 & 0 & & & \\
\hline 4 & -70 & 0 & 0 & 0 & 0 & 0 & 0 & 0 & & & \\
\hline 5 & 10 & 0 & 0 & 1.8 & 1.8 & 3.6 & 0 & 3.6 & 11.14 & 10.83 & G2 \\
\hline 6 & 20 & 0 & 0 & 9.6 & 9.6 & 19.2 & 0 & 19.2 & 10.95 & 10.38 & $\mathrm{G} 2$ \\
\hline 7 & 40 & 15 & 15 & 9.6 & 0 & 39.6 & 9.6 & 30 & 10.27 & 10.25 & $\mathrm{G} 2, \mathrm{G} 1$ \\
\hline 8 & 40 & 15 & 15 & 0 & 9.6 & 39.6 & 9.6 & 30 & 10.41 & 10.01 & $\mathrm{G} 2, \mathrm{G} 1$ \\
\hline 9 & 30 & 0 & 0 & 16.8 & 9.6 & 26.4 & 0 & 26.4 & 10.57 & 10.36 & G2 \\
\hline 10 & 30 & 0 & 0 & 9.6 & 16.8 & 26.4 & 0 & 26.4 & 10.57 & 10.36 & G2 \\
\hline 11 & -10 & 0 & 0 & 0 & 0 & 0 & 0 & 0 & & & \\
\hline 12 & -20 & 0 & 0 & 0 & 0 & 0 & 0 & 0 & & & \\
\hline 13 & 50 & 15 & 15 & 9.6 & 9.6 & 49.2 & 19.2 & 30 & 10.17 & 10.02 & $\mathrm{G} 2, \mathrm{G} 1$ \\
\hline 14 & 50 & 15 & 15 & 9.6 & 9.6 & 49.2 & 19.2 & 30 & 10.17 & 10.02 & $\mathrm{G} 2, \mathrm{G} 1$ \\
\hline 15 & 10 & 0 & 0 & 0 & 0 & 0 & 0 & 0 & & & \\
\hline 16 & 10 & 0 & 0 & 0 & 0 & 0 & 0 & 0 & & & \\
\hline
\end{tabular}

Unit of power is in $\mathrm{kW}$ and Price is in $(\mathrm{c} / \mathrm{kWh})$ 
Table 5 Real-time generation and load information without DR participation (Stage-3)

\begin{tabular}{|c|c|c|c|c|c|c|c|c|c|}
\hline \multirow[t]{2}{*}{ DI } & \multicolumn{2}{|c|}{ Sellers $^{\mathrm{a}}$} & \multicolumn{2}{|c|}{ Buyers $^{\mathrm{a}}$} & \multirow[t]{2}{*}{$\Delta \mathrm{P}^{a}$} & \multirow[t]{2}{*}{$\mathrm{MCP}^{\mathrm{b}}$} & \multicolumn{2}{|c|}{ Inelastic load $^{\mathrm{a}}$} & \multirow[t]{2}{*}{$\Delta P_{r}^{\mathrm{a}}$} \\
\hline & SA1 & SA2 & BA1 & BA2 & & & BA1 & BA2 & \\
\hline 1 & 60 & 50 & 90 & 90 & -70 & 12.35 & 56.4 & 60 & -6.4 \\
\hline 2 & 50 & 60 & 90 & 90 & -70 & 12.35 & 56.4 & 60 & -6.4 \\
\hline 3 & 50 & 50 & 90 & 90 & -80 & 12.56 & 56.4 & 60 & -16.4 \\
\hline 4 & 60 & 50 & 90 & 90 & -70 & 12.35 & 56.4 & 60 & -6.4 \\
\hline 5 & 70 & 70 & 60 & 60 & 20 & 10.98 & 60 & 60 & 20 \\
\hline 6 & 80 & 60 & 60 & 60 & 20 & 10.98 & 60 & 60 & 20 \\
\hline 7 & 80 & 70 & 50 & 55 & 45 & 10.54 & 50 & 55 & 45 \\
\hline 8 & 80 & 80 & 50 & 60 & 50 & 10.50 & 50 & 60 & 50 \\
\hline 9 & 80 & 70 & 50 & 60 & 40 & 10.64 & 50 & 60 & 40 \\
\hline 10 & 90 & 50 & 50 & 60 & 30 & 10.80 & 50 & 60 & 30 \\
\hline 11 & 60 & 60 & 60 & 80 & -20 & 11.62 & 60 & 80 & -20 \\
\hline 12 & 70 & 60 & 70 & 80 & -20 & 11.60 & 70 & 80 & -20 \\
\hline 13 & 80 & 70 & 60 & 60 & 30 & 10.83 & 60 & 60 & 30 \\
\hline 14 & 70 & 70 & 50 & 55 & 35 & 10.69 & 50 & 55 & 35 \\
\hline 15 & 70 & 60 & 70 & 80 & -20 & 11.60 & 70 & 80 & -20 \\
\hline 16 & 70 & 60 & 70 & 50 & 10 & 11.13 & 70 & 50 & 10 \\
\hline
\end{tabular}

${ }^{a}$ Unit of power in $\mathrm{kW}$

b Unit of Price in $(\mathrm{c} / \mathrm{kWh})$

Table 6 Scheduling of DG contracts (Stage-3)

\begin{tabular}{|c|c|c|c|c|c|c|c|c|c|c|c|}
\hline \multirow[t]{2}{*}{ DI } & \multirow[t]{2}{*}{$\Delta P_{r}^{a}$} & \multicolumn{4}{|c|}{ Final schedule ${ }^{\mathrm{a}}$} & \multirow[t]{2}{*}{$\Sigma$ DR Load $^{\text {a }}$} & \multicolumn{2}{|c|}{ SPA bids ${ }^{b}$} & \multirow[t]{2}{*}{ Auction winner } & \multicolumn{2}{|c|}{ Contract $^{a}$} \\
\hline & & BA11 & BA12 & $B A 21$ & $B A 22$ & & SA1 & SA2 & & SA1 & SA2 \\
\hline 1 & -6.4 & 0 & 0 & 0 & 0 & 0 & & & & 0 & 0 \\
\hline 2 & -6.4 & 0 & 0 & 0 & 0 & 0 & & & & 0 & 0 \\
\hline 3 & -16.4 & 0 & 0 & 0 & 0 & 0 & & & & 0 & 0 \\
\hline 4 & -6.4 & 0 & 0 & 0 & 0 & 0 & & & & 0 & 0 \\
\hline 5 & 20 & 9.6 & 9.6 & 0 & 0 & 19.2 & 11.14 & 10.83 & G2 & 0 & 19 \\
\hline 6 & 20 & 9.6 & 9.6 & 0 & 0 & 19.2 & 10.95 & 10.38 & $\mathrm{G} 2$ & 0 & 19.2 \\
\hline 7 & 45 & 9.6 & 1.8 & 15 & 15 & 41.4 & 10.27 & 10.25 & $\mathrm{G} 2, \mathrm{G} 1$ & 11.4 & 30 \\
\hline 8 & 50 & 9.6 & 9.6 & 15 & 15 & 49.2 & 10.41 & 10.01 & $\mathrm{G} 2, \mathrm{G} 1$ & 19.2 & 30 \\
\hline 9 & 40 & 16.8 & 16.8 & 0 & 0 & 33.6 & 10.57 & 10.36 & $\mathrm{G} 2, \mathrm{G} 1$ & 3.6 & 30 \\
\hline 10 & 30 & 9.6 & 16.8 & 0 & 0 & 26.4 & 10.57 & 10.36 & G2 & 0 & 26.4 \\
\hline 11 & -20 & 0 & 0 & 0 & 0 & 0 & & & & 0 & 0 \\
\hline 12 & -20 & 0 & 0 & 0 & 0 & 0 & & & & 0 & 0 \\
\hline 13 & 30 & 0 & 0 & 15 & 15 & 30 & 10.17 & 10.02 & G2 & 0 & 30 \\
\hline 14 & 35 & 1.8 & 1.8 & 15 & 15 & 33.6 & 10.17 & 10.02 & $\mathrm{G} 2, \mathrm{G} 1$ & 3.6 & 30 \\
\hline 15 & -20 & 0 & 0 & 0 & 0 & 0 & & & & 0 & 0 \\
\hline 16 & 10 & 1.8 & 1.8 & 0 & 0 & 3.6 & 11.14 & 10.83 & G2 & 0 & 3.6 \\
\hline
\end{tabular}

${ }^{\text {a }}$ Unit of power in $\mathrm{kW}$

${ }^{\mathrm{b}}$ Unit of Price in $(\mathrm{c} / \mathrm{kWh})$

Table 8 shows the comparison among two methods for the case study data given in Tables 1, 2, 3, 4 and 5. From the comparison, it can be observed that the elastic loads or DR loads are served at lesser price by adopting the proposed incentive mechanism than adopting the method given [18].
In the proposed mechanism the shares of the responsive endusers are dynamic due to their incentive usage, whereas in [18] the ranks of the end-users are kept static irrespective of their incentive usage. 
Table 7 Finalized schedule of sellers, buyers and incentive metric and load served details (Stage-4)

\begin{tabular}{|c|c|c|c|c|c|c|c|c|c|c|c|c|}
\hline \multirow[t]{2}{*}{ DI } & \multicolumn{2}{|c|}{ Sellers ${ }^{\mathrm{a}}$} & \multicolumn{2}{|c|}{ Buyers $^{\mathrm{a}}$} & \multicolumn{2}{|l|}{$I M_{i}$} & \multicolumn{2}{|c|}{ Generation share $^{\mathrm{a}}$} & \multicolumn{2}{|c|}{ Load served in $\mu \mathrm{g}^{\mathrm{a}}$} & \multicolumn{2}{|c|}{ Load served by UGA } \\
\hline & SA1 & SA2 & BA1 & BA2 & BA1 & BA2 & BA1 & BA2 & BA1 & BA2 & BA1 & BA2 \\
\hline 1 & 60 & 50 & 56.4 & 60 & 0.5 & 0.5 & 55 & 55 & 55 & 55 & 1.4 & 5 \\
\hline 2 & 50 & 60 & 56.4 & 60 & 0.5 & 0.5 & 55 & 55 & 55 & 55 & 1.4 & 5 \\
\hline 3 & 50 & 50 & 56.4 & 60 & 0.5 & 0.5 & 50 & 50 & 50 & 50 & 6.4 & 10 \\
\hline 4 & 60 & 50 & 56.4 & 60 & 0.5 & 0.5 & 55 & 55 & 55 & 55 & 1.4 & 5 \\
\hline 5 & 70 & 51 & 79.2 & 60 & 0.5 & 0.5 & & & 79.2 & 60 & 0 & 0 \\
\hline 6 & 80 & 41 & 79.2 & 60 & 0.5 & 0.5 & & & 79.2 & 60 & 0 & 0 \\
\hline 7 & 69 & 40 & 61.4 & 85 & 0.54 & 0.46 & & & 61.4 & 85 & 0 & 0 \\
\hline 8 & 61 & 50 & 69.2 & 90 & 0.5 & 0.5 & & & 69.2 & 90 & 0 & 0 \\
\hline 9 & 76 & 40 & 83.6 & 60 & 0.48 & 0.52 & & & 83.6 & 60 & 0 & 0 \\
\hline 10 & 90 & 24 & 76.4 & 60 & 0.52 & 0.48 & & & 76.4 & 60 & 0 & 0 \\
\hline 11 & 60 & 60 & 60.0 & 80 & 0.56 & 0.44 & 66.67 & 53.33 & 60 & 53.33 & 0 & 26.67 \\
\hline 12 & 70 & 60 & 70.0 & 80 & 0.57 & 0.43 & 74.29 & 55.71 & 70 & 55.71 & 0 & 24.29 \\
\hline 13 & 80 & 40 & 60.0 & 90 & 0.6 & 0.4 & & & 60 & 90 & 0 & 0 \\
\hline 14 & 66 & 40 & 53.6 & 85 & 1 & 0 & & & 53.6 & 85 & 0 & 0 \\
\hline 15 & 70 & 60 & 70.0 & 80 & 1 & - & 130 & 0 & 70 & 0 & 0 & 80 \\
\hline 16 & 70 & 56 & 73.6 & 50 & - & - & & & 73.6 & 50 & 0 & 0 \\
\hline
\end{tabular}

${ }^{\mathrm{a}}$ Unit of power in $\mathrm{kW}$

Table 8 Cost comparison and savings

\begin{tabular}{lcc}
\hline Comparison & BA1 & BA2 \\
\hline Case 1: Without DR (c) & 417 & 372 \\
Case 2: Proposed Method (c) & 356 & 302 \\
Case 3: Existing Method (c) & 383 & 338 \\
Savings: Case 2 vs 1 (c) & 61 & 70 \\
Savings: Case 3 vs 1 (c) & 34 & 70 \\
Savings: Case 3 vs 2 (c) & 28 & 35 \\
\hline
\end{tabular}

\section{Conclusion}

In this paper, a new incentive mechanism and DR strategy have been proposed to accommodate and encourage PEVs' user participation in DR programs of smart microgrid. The proposed 'priority rewarding' incentive mechanism encourages electricity consumers to continuously participate in DR programs and distinctly captures the flexibility offered by various DR loads viz. adjustable, shiftable and curtailable. An incentive metric is assigned to monitor the DR enrolments and incentive usage. The DR strategy proposed to handle responsive loads is successful in obtaining bilateral contracts between DR aggregator and DGs. The applicability of SPA is tested for obtaining DG contracts to capture its advantage in offering reduced auction clearance time. From the case study results, it can be concluded that the proposed incentive mechanism is successful in motivating the consumers to get enrolled in DR programs.
Acknowledgments This work is supported by IRCC, IIT Bombay (09IRCC040) and Department of Science and Technology (14DSTID002, INT/NL/SEG/P-1/2014).

\section{References}

1. Ferruzzi, G., Graditi, G., Rossi, F., Russo, A.: Optimal operation of a residential microgrid: The role of demand side management. Intell. Ind. Syst. 1(1), 61-82 (2015)

2. Ali, M., Safdarian, A., Lehtonen, M.: Demand response potential of residential hvac loads considering users preferences. In: Innovative Smart Grid Technologies Conference Europe (ISGT-Europe), 2014 IEEE PES. pp. 1-6 (2014)

3. Narimani, M., Joo, J.-Y., Crow, M.: The effect of demand response on distribution system operation. In: Power and Energy Conference at Illinois (PECI), 2015 IEEE. pp. 1-6 (2015)

4. Siano, P.: Demand response and smart grids-a survey. Renew Sustain Energy Rev 30, 461-478 (2014)

5. Nunna, H., Doolla, S.: Demand response in smart microgrids. In: Innovative Smart Grid Technologies - India (ISGT India), 2011 IEEE PES. pp. 131-136 (2011)

6. Sesetti, A., Battula, S., Nunna, H., Doolla, S.: Intelligent agent framework for demand response aggregation in smart microgrids. In: Industrial Electronics Society, IECON 2014 - 40th Annual Conference of the IEEE. pp. 3549-3555 (2014)

7. Taheri, N., Entriken, R., Ye, Y.: A dynamic algorithm for facilitated charging of plug-in electric vehicles. Smart Grid IEEE Trans. 4(4), 1772-1779 (2013)

8. Zakariazadeh, A., Jadid, S., Siano, P.: Integrated operation of electric vehicles and renewable generation in a smart distribution system. Energy Convers. Manag. 89, 99-110 (2015)

9. Honarmand, M., Zakariazadeh, A., Jadid, S.: Integrated scheduling of renewable generation and electric vehicles parking lot in a smart microgrid. Energy Convers. Manag. 86, 745-755 (2014) 
10. Honarmand, M., Zakariazadeh, A., Jadid, S.: Self-scheduling of electric vehicles in an intelligent parking lot using stochastic optimization. J Frankl. Inst. 352(2), 449-467 (2015)

11. Ul-Haq, A., Cecati, C., Strunz, K., Abbasi, E.: Impact of electric vehicle charging on voltage unbalance in an urban distribution network. Intell. Ind. Syst. 1(1), 51-60 (2015)

12. Moghbel, M., Masoum, M. A., Fereidouni, A.: Decentralize coordinated charging of plug-in electric vehicles in unbalanced residential networks to control distribution transformer loading, voltage profile and current unbalance. Intell. Ind. Syst. 1(1), 141-151 (2015)

13. Sarker, M., Ortega-Vazquez, M., Kirschen, D.: Optimal coordination and scheduling of demand response via monetary incentives. Smart Grid IEEE Trans. 6(3), 1341-1352 (2015)

14. Mallette, M., Venkataramanan, G.: Financial incentives to encourage demand response participation by plug-in hybrid electric vehicle owners. In: Energy Conversion Congress and Exposition (ECCE), 2010 IEEE. pp. 4278-4284 (2010)

15. Khajavi, P., Abniki, H., Arani, A.: The role of incentive based demand response programs in smart grid. In: Environment and Electrical Engineering (EEEIC), 2011 10th International Conference on. pp. 1-4 (2011)

16. Shu, H., Yu, R., Rahardja, S.: Dynamic incentive strategy for voluntary demand response based on tdp scheme. In: Signal Information Processing Association Annual Summit and Conference (APSIPA ASC), 2012 Asia-Pacific. pp. 1-6 (2012)
17. Zhong, H., Xie, L., Xia, Q.: Coupon incentive-based demand response: theory and case study. Power Syst.IEEE Trans. 28(2), 1266-1276 (2013)

18. Nunna, H., Doolla, S.: Responsive end-user-based demand side management in multimicrogrid environment. IEEE Trans. Ind. Inform. 10(2), 1262-1272 (2014)

19. McArthur, S.D., Davidson, E.M., Catterson, V.M., Dimeas, A.L., Hatziargyriou, N.D., Ponci, F., Funabashi, T.: Multi-agent systems for power engineering applications-part i: concepts, approaches, and technical challenges. Power Syst. IEEE Trans. 22(4), 17431752 (2007)

20. Suli, Z., Zhongjing, M., Xiangdong, L.: Optimal microgrid economic operations under progressive second price auction mechanisms. In: Control Conference (CCC), 2013 32nd Chinese. pp. 8891-8896 (2013)

21. "Charging," http://my.teslamotors.com/roadster/charging, [Online; Accessed 15 Dec 2014] 